\title{
Reseña del libro. Didáctica de las Ciencias Experimentales en Educación Primaria
}

\author{
Autores. Ana Rivero, Rosa Martín del Pozo, Emilio Solís y Rafael Porlán \\ Editorial: Síntesis \\ Lugar de edición: Madrid. España \\ Número de páginas: 268
}

Año: 2017

ISBN. 978-84-9171-062-2

ÍNDICE

\section{Prólogo}

Parte I. Fundamentos

1. Didáctica de las ciencias: mucho más que la manera de enseñar ciencias

2.Naturaleza de la ciencia

3. Finalidades de la educación científica

4. Enseñar y aprender ciencias en educación primaria

Parte II). El diseño de la práctica

5. Las ideas de los alumnos en ciencias

6. Los contenidos escolares de ciencias

7. Metodología de enseñanza

8. Evaluación de la enseñanza y aprendizaje de las ciencias

Parte III. La práctica y la reflexión

9. La práctica de la investigación escolar en ciencias

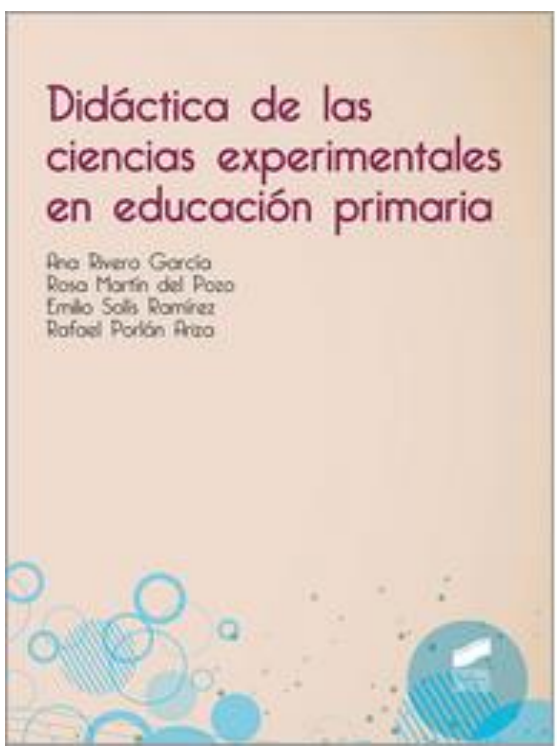

10. Aprender a enseñar ciencias

Bibliografía

Para enseñar ciencias en Primaria no basta con saber los contenidos de ciencias. Ni con saber unas cuantas técnicas para enseñar. Ni con tener ciertas características personales (paciencia, empatía, amabilidad,...) Para enseñar ciencias en Primaria hay que estar dispuestos a poner en cuestión los estereotipos tradicionales sobre la ciencia y sobre su enseñanza y aprendizaje, entre otras razones porque todos hemos pasado por el sistema escolar como estudiantes y nos hemos impregnado de ellos sin ser conscientes. Estos estereotipos hay que compararlos críticamente con las teorías y las prácticas innovadoras, fruto de la investigación didáctica y de la experiencia docente de calidad. Los cambios que surjan de este contraste hay que ponerlos en práctica, analizarlos y mejorarlos progresivamente. Así se aprende a enseñar ciencias de manera profesional. Ese es el proceso que los autores pretendemos facilitar. Eso es lo que hacemos en nuestras clases de la Facultad de Educación y es el sentido básico de este manual. Para ello nos apoyamos en los avances de la Didáctica de las Ciencias como disciplina que ofrece propuestas fundamentadas de intervención para mejorar la educación científica de la ciudadanía y la formación del profesorado. 
El manual que presentamos es producto de nuestra ya dilatada experiencia como profesores de Didáctica de las Ciencias en la formación inicial de maestros y como investigadores en los muchos proyectos de investigación sobre el conocimiento profesional necesario para enseñar ciencias y las estrategias y recursos para la formación de maestros en este ámbito. Además, los autores pertenecemos a la red IRES (Innovación y Renovación Escolar), junto con profesores de todos los niveles educativos, por lo que consideramos que este manual es también fruto de nuestras experiencias y debates conjuntos (www.redires.net).

El manual tiene una orientación práctica y se concibe como un instrumento para facilitar el aprendizaje de la enseñanza de las ciencias en Primaria, y creemos que por ello puede ser de utilidad para maestros (en formación y en activo) y para formadores en el área de Didáctica de las Ciencias. Además, muchas de las aportaciones que se hacen pueden ser de utilidad en la formación inicial del profesorado de ciencias de Secundaria.

Está organizado en 10 capítulos y se diferencian tres partes. No obstante, el primer capítulo tiene un carácter especial ya que intenta mostrar el enorme potencial de la Didáctica de las Ciencias para la innovación en el aula y la formación de los maestros, superando la idea de que la didáctica se ocupa solo del cómo enseñar ciencias al alumnado. En la primera parte, los capítulos 2 (sobre la naturaleza de la ciencia), 3 (sobre las finalidades de la educación científica) y 4 (sobre el proceso de enseñanza y aprendizaje de las ciencias), permiten reflexionar sobre estas cuestiones básicas que fundamentan el diseño de la práctica de la enseñanza de las ciencias. En la segunda parte, los capítulos 5 (sobre las ideas de los alumnos en ciencias), 6 (sobre los contenidos escolares de ciencias), 7 (sobre la metodología de enseñanza de las ciencias) y 8 (sobre la evaluación en ciencias), pretenden acompañar al lector en el proceso de diseño de un plan de enseñanza de las ciencias a partir de su propia propuesta, que se irá analizando y contrastando con otros planteamientos didácticos que tienen como referencia el modelo de investigación escolar en ciencias. En la tercera parte, el capítulo 9 recoge la voz de los maestros que enseñan ciencias en sus aulas por investigación escolar y reflexionan sobre ello. Se trata de poner de manifiesto que la investigación escolar en ciencias es posible y tiene ventajas y también dificultades para su puesta en práctica. Este acercamiento a la práctica también nos permitirá reflexionar sobre las relaciones teoría-práctica. Finalmente, en el capítulo 10 se pretende reflexionar sobre todo el proceso formativo seguido, mediante la descripción de lo que, como formadores de futuros maestros, realizamos en las clases de Didáctica de las Ciencias, y que, salvadas las diferencias, hemos pretendido recoger con los mismos planteamientos en este manual.

Los capítulos se inician con un breve comentario acerca del interés de la temática a tratar y lo que se pretende abordar. A continuación, se plantea una reflexión inicial sobre dicha temática, proponiendo cuestiones que el lector puede responder y después ir contrastando a lo largo del capítulo. En sus diferentes apartados se incluyen un total de 66 actividades concretas, textos de lectura, legislación, esquemas, guiones de reflexión, guiones de análisis, enlaces web...etc. De especial importancia es la actividad en la que se trata de seleccionar un tema de ciencias para Primaria e ir elaborando una propuesta de enseñanza. Dicha propuesta se irá "mejorando" a lo largo de los diferentes capítulos. Para finalizar, se propone una síntesis del contenido del capítulo, así como unas referencias bibliográficas básicas. El manual termina con una bibliografía básica en Didáctica de las Ciencias, así como con una relación de los sitios 
web más utilizados por nosotros en la formación de maestros.

Se trata pues de una propuesta formativa para aprender a enseñar ciencias que participa del espíritu innovador que quiere mejorar la práctica, contribuyendo a hacer frente al principal reto de la Didáctica de las Ciencias: superar las dificultades que plantea un modelo transmisivo de enseñanza que genera fracaso escolar y profesional mediante la extensión de un modelo de investigación escolar en la enseñanza de las ciencias y de investigación profesional en la formación del profesorado de ciencias.

Queremos dedicar este libro a los formadores que enseñan a enseñar ciencias -con el mismo modelo o enfoque con el que pretenden que los futuros maestros enseñen ciencias a sus alumnos: aquí el medio es el mensaje. Muchos futuros maestros que participan en nuestras clases nos lo dicen: "lo que nosotros hacemos en clase es como lo tendríamos que hacer con los alumnos de Primaria, ¿no?" Sí claro, salvando las distancias. Es decir, la adopción del principio de investigación como un principio didáctico y formativo de síntesis. Así, la idea de investigación no sólo debe estar presente en el modelo de enseñanza de las ciencias (Inquiry-based Science Education) sino también en el modelo de la formación de maestros (Inquiry-based Science Teacher Education).

Los autores

Referencia: Rivero, A., Martín del Pozo, R., Solís, E. y Porlán, R. (2017). Didáctica de las Ciencias Experimentales en Educación Primaria. Madrid: Editorial Síntesis

Contacto. arivero@us.es, rmartin@edu.ucm.es, esolis@us.es, rporlan@us.es 